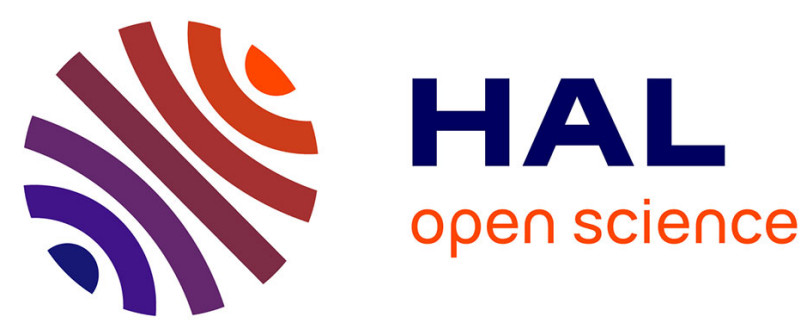

\title{
Evaluation of Multicasting Schemes based on Joint Multiple Description and Network Coding
}

\author{
Hamlet Jesse Medina-Ruiz, Lana Iwaza, Michel Kieffer, Beatrice \\ Pesquet-Popescu, Khaldoun Al Agha
}

\section{- To cite this version:}

Hamlet Jesse Medina-Ruiz, Lana Iwaza, Michel Kieffer, Beatrice Pesquet-Popescu, Khaldoun Al Agha. Evaluation of Multicasting Schemes based on Joint Multiple Description and Network Coding. IEEE WCNC 2012, Apr 2012, Paris, France. pp.1160-1664, 10.1109/WCNC.2012.6214049 . hal-00727782

\section{HAL Id: hal-00727782}

https://hal-centralesupelec.archives-ouvertes.fr/hal-00727782

Submitted on 4 Sep 2012

HAL is a multi-disciplinary open access archive for the deposit and dissemination of scientific research documents, whether they are published or not. The documents may come from teaching and research institutions in France or abroad, or from public or private research centers.
L'archive ouverte pluridisciplinaire HAL, est destinée au dépôt et à la diffusion de documents scientifiques de niveau recherche, publiés ou non, émanant des établissements d'enseignement et de recherche français ou étrangers, des laboratoires publics ou privés. 


\title{
Evaluation of Multicasting Schemes based on
}

\section{Joint Multiple Description and Network Coding}

\author{
Hamlet Medina Ruiz ${ }^{* \dagger}$, Lana Iwaza*, Michel Kieffer*†, Béatrice Pesquet-Popescu ${ }^{\dagger}$ and Khaldoun Al Agha ${ }^{\ddagger}$ \\ ${ }^{*}$ L2S - CNRS - SUPELEC - Univ Paris-Sud \\ 3 rue Joliot-Curie, 91192 Gif-sur-Yvette, France \\ †Institut Télécom, Télécom ParisTech and CNRS LTCI \\ Signal and Image Processing Department, \\ 46 rue Barrault, 75634 Paris Cedex 13, France \\ ${ }^{\ddagger}$ LRI - CNRS - Univ Paris-Sud \\ 91405 Orsay, France
}

\begin{abstract}
This paper considers a multicast scenario and compares the average reception quality obtained when combining multiple description coding (MDC) and network coding (NC). Plain (single description) network coding (NC-SDC) serves as reference.

In the considered scenario, a single source is multicast to several receivers with various channel conditions. Contrary to a NC-SDC scheme, unable to recover the coded packets when not enough combinations of packets have been received, $\mathrm{NC}$ of $\mathrm{MDC}$ packets allows a more progressive quality improvement with the number of received packets, and a reduction of the effect of the quantization noise when MDC is performed via frame expansion before quantization.

Considering a probability distribution for the bit transition probability during transmission to any user in the multicast group, the expected signal-to-noise ratio is evaluated. Performance comparisons are made for various error distributions, field sizes, and MDC methods (via frame expansion and correlating transform).
\end{abstract}

\section{INTRODUCTION}

The quality of reception in any multimedia transmission in cellular networks or between neighbors in ad-hoc wireless networks is highly dependent on the channel conditions between the receiver and its base station, or between neighbors.

Efficient coding schemes have to be designed in such a way that they adapt to the various channel conditions of the different users: users with better channel conditions should get a content with an improved quality, without penalizing those with worse conditions, the maximum of which should still be able to get the transmitted content with a decent quality.

Scalable or Layered Coding (LC) techniques [1] is one way to implement such adaptive systems. In LC, transmitted data are partitioned into several quality layers with corresponding priority. The decoding quality enhances with the number of layers received. The combination of this technique with a priority encoding transmission (PET) can increase its efficiency. However, with LC, receiving a low priority layer is useless as long as all higher priority layers have not been received.
A way to overcome this drawback is to use Multiple Description Coding (MDC) [2]. Data are partitioned into equally important descriptions, and the quality of reception gracefully improves with the number of received descriptions. The price to be paid is some additional redundancy introduced during coding. Routing of MDC data may be optimized using rainbow network coding [3]. The reconstruction quality is maximized by optimizing the combinations of descriptions associated to each link. Nevertheless, this approach is centralized and requires a perfect knowledge of the network topology.

Network coding (NC) [4], in multicast scenarios, may achieve the max-flow capacity between the source and each destination node [5]. Practical random NC schemes have been proposed, see, e.g., [6]. NC is quite robust to packet losses, as long as enough coded packets are received. When not enough packets are obtained, no reconstruction is possible. Joint source-network coding approaches have been proposed recently in order to get the best out of MDC and NC. Concatenated MDC and NC have been first proposed in [7], where a network code and an outer PET code are concatenated to allow users with bad channel conditions to be able to decode the most important packets even if the reception of all packets is not guaranteed. In [8], approximate decoding is performed when not enough packets are received, by exploiting existing correlations between transmitted packets. In [9], a coding scheme is proposed combining NC and MDC, where descriptions are generated either via frame expansion (NC-MDC-F), or via a correlating transform (NC-MDC-T). A good robustness against packet losses is observed in both cases. While NC-MDC-T is tolerant to more erasures, NCMDC-F provides a better signal-to-noise ratio (SNR) when the number of lost packets is small thanks to the reduction of a part of the quantization noise. Moreover, NC-MDC-F is able in some cases to provide a reconstruction even if the number of losses is higher than the number of redundant packets introduced during frame expansion. The price to be paid is an increased computational complexity, since a mixed 
integer quadratic program has to be solved.

This paper evaluates the performance of both NC-MDC approaches and compares them to a scenario where only $\mathrm{NC}$ is performed (NC-SDC). These schemes are briefly recalled in Section II. We assume that the same number of potentially corrupted packets are available to each receiver. These packets are assumed to have passed through a binary symmetric channel (BSC) with transition probability $\varepsilon$ distributed according to some probability density function (pdf) $f(\varepsilon)$. This allows us to characterize for the three coding schemes the average SNR among receivers for various $f(\varepsilon)$, as detailed in Section III. Simulation results are described in Section IV, before drawing some conclusions and introducing future research directions in Section V.

\section{CODING SchemeS}

Assume that a source $S$ has to transmit some realization $x \in \mathbb{R}^{k}$ of a random vector to $N$ receiver nodes. Three types of coding schemes are considered. The resulting models of the transmission chain between the source and a typical receiver are now presented. These models may correspond to various communication networks such as a (possibly relay-assisted) cellular network or a wireless ad-hoc network.

\section{A. Scenario 1: single description network coding (NC-SDC)}

In this scenario, each entry of the source vector $x$ is quantized using a $q$-level uniform scalar quantizer to get a vector of quantized indexes $z \in \mathbb{F}_{q}^{k}$, where $\mathbb{F}_{q}$ is the Galois field with $q$ elements. Each quantized index is put in a separate packet. Then $n \geqslant k$ random linear combinations of packets over $\mathbb{F}_{q}^{k}$ are transmitted. Further combinations may be performed within the network. A receiver then gets $m \leqslant n$ packets $p \in \mathbb{F}_{q}^{m}$, see Figure 1. The effect of the network and the various network coding operations is modeled by the network coding matrix $A$ linking $z$ and $p$ as follows:

$$
p=A z \text {. }
$$

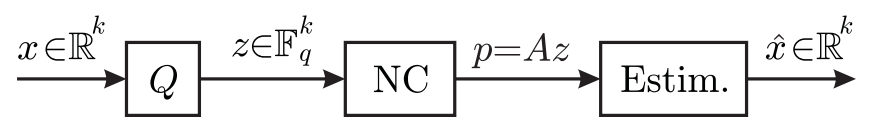

Fig. 1. Block diagram of the NC-SDC scenario

The estimate $\widehat{x}$ of $x$ at the receiver side requires that $A$ is full rank $k$, in which case $z$ is obtained via Gaussian elimination and $\widehat{x}$ via inverse quantization of $z$. If $A$ is of rank less than $k$, no estimate better than the mean value of $x$ may be obtained.

\section{B. Scenario 2: MDC via correlating transform (NC-MDC-T)}

Again, $x$ is quantized to get quantization indexes $y \in \mathbb{F}_{q}^{k}$. Then, a full-rank $k$ correlating transform $T \in \mathbb{F}_{q}^{n \times k}$ is applied to $y$ to get a vector $z=T y \in \mathbb{F}_{q}^{n}$. Entries of $z$ are again put in separate packets which are network coded, and $p \in \mathbb{F}_{q}^{m}$ represents the received packets, see (1) and Figure 2.

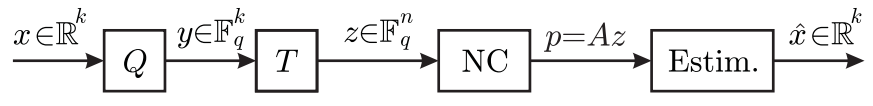

Fig. 2. Block diagram of the MDC scheme via correlating transform (NCMDC-T)

Since $T \in \mathbb{F}_{q}^{n \times k}$ is of full rank $k$, there exists a parity-check matrix $H \in \mathbb{F}_{q}^{(n-k) \times n}$ of rank $n-k$ such that $H z=0$ for all $z$ such that there exists $y \in \mathbb{F}_{q}^{k}$ with $z=T y$. This property may be used at receiver side to estimate $\widehat{x}$ from $p$. Provided that the matrix

$$
A^{\prime}=\left(\begin{array}{c}
A \\
H
\end{array}\right)
$$

is of rank $n, z$ may again be obtained via Gaussian elimination, and $\widehat{x}$ via inverse quantization, see [8], [9]. When $A^{\prime}$ is of rank less than $n$, again, no better estimate than the mean value of $x$ may be obtained.

\section{Scenario 3: MDC via frame expansion (NC-MDC-F)}

Here, the redundancy is introduced via a frame expansion matrix $F \in \mathbb{R}^{n \times k}$, producing the vector $y=F x \in \mathbb{R}^{n}$ with $n \geqslant k$. Each entry of $y$ is then quantized with a $q$-level uniform scalar quantizer to get a vector of indexes $z \in F_{q}^{n}$. The entries of $z$ are then processed as in Scenarios 1 and 2 .

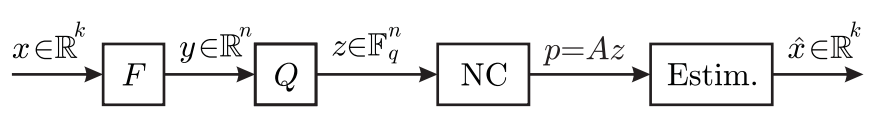

Fig. 3. Block diagram of the MDC scheme via frame expansion (NC-MDCF)

At the receiver side, $p$ and the fact that $x$ has been expanded may be exploited to obtain $\widehat{x}$. In [9], this problem is cast in the framework of mixed integer quadratic programming. The problem may then be modeled, e.g., with AMPL [10] and solved, e.g., using CPLEX [11].

Remark 1: In the previous scenarios, packets contain only a single quantized sample. In what follows, we assume that the source waits for $\ell$ realizations of the source vector before starting transmission. Packets of $\ell$ samples are then transmitted, which improves the payload length over total packet length ratio.

\section{EXPECTED SIGNAL-TO-NOISE RATIO FOR A GIVEN RECEIVER}

When considering a single receiver, with known channel characteristics, there is no need to perform any joint coding scheme, a separate optimization of the data compression and transmission being optimal, see [12].

In the considered multicast scenario, the answer is not so clear. The aim of this paper is thus to evaluate the performance of the three previously-introduced coding schemes as a function of the size $q$ of $\mathbb{F}_{q}$ and for different channel models. 


\section{A. Hypotheses}

The number of network-coded packets (size of the generation) is $g$. For NC-SDC one has $g=k$ whereas for NC-MDC$\mathrm{T}$ and NC-MDC-F, $g=n$. It is assumed that each of the $N$ receivers obtains $n$ noisy packets from which an estimate of $x$ has to be evaluated. The packets are assumed to have passed through a binary symmetric channel (BSC) with transition probability $\varepsilon$ distributed according to some probability density function (pdf) $f(\varepsilon)$, identical for all the receivers. This BSC model allows us to account for the packet length, which is not the same in the three considered scenarios.

The fact that all receivers have obtained the same number of noisy packets is quite realistic in a cellular network. It is more questionable in an ad-hoc network, where the min-cut is likely not to be the same between the source and all users.

\section{B. Average signal-to-noise ratio}

The average SNR observed by a given receiver depends on the number of received packets as well as the size of the Galois field $q$. For a given user, whatever the considered scenario, one has to determine the probability mass function (pmf) $P_{\Gamma}(\gamma \mid g, n, q)$ of the number $\gamma$ independent packets received, i.e., the pmf of the rank of $A$, given that $g$ packets were combined, $n$ noisy combinations were received, and that the size of the Galois field is $q$.

If $i$ is the number of error-free packets received, one gets

$$
\begin{aligned}
P_{\Gamma}(\gamma \mid g, n, q) & =\sum_{i=\gamma}^{n} P(\gamma, i \mid g, n, q) \\
& =\sum_{i=\gamma}^{n} P(\gamma \mid i, g, n, q) P(i \mid g, n, q) \\
& =\sum_{i=\gamma}^{n} P_{R}(\gamma \mid i, g, q) P_{C}(i \mid g, n, q) .
\end{aligned}
$$

In (3), $P_{C}(i \mid g, n, q)$ is the probability of getting $i$ noisefree (Clean) packets (this probability depends on $f(\varepsilon)$ ) and $P_{R}(\gamma \mid i, g, q)$ is the probability of having $\gamma$ informative packets among the $i$ received packets without errors (this is the probability that the rank of $A$ is $\gamma$ ).

To evaluate $P_{C}(i \mid g, n, q)$, one has to introduce $f(\varepsilon)$ as follows

$$
\begin{aligned}
P_{C}(i \mid g, n, q) & =\int P(i, \varepsilon \mid g, n, q) d \varepsilon \\
& =\int P(i \mid g, n, q, \varepsilon) f(\varepsilon \mid g, n, q) d \varepsilon \\
& =\int P(i \mid g, n, q, \varepsilon) f(\varepsilon) d \varepsilon,
\end{aligned}
$$

since $f(\varepsilon)$ does not depend neither on $n$, nor on $q$.

Then

$$
P(i \mid g, n, q, \varepsilon)=\left(\begin{array}{c}
n \\
i
\end{array}\right)(1-\varepsilon)^{i L}\left(1-(1-\varepsilon)^{L}\right)^{n-i}
$$

indicates the probability of receiving $i$ packets without errors among $n$ received packets sent for a realization $\varepsilon$ of the BSC transition probability. In (5), $L=\left(g+\ell+\ell_{\text {seq }}+\ell_{\text {crc }}\right)\left\lceil\log _{2} q\right\rceil$ represents the total length in bits of a packet. $\ell_{\text {seq }}$ and $\ell_{\text {crc }}$ stand for the number of symbols in $\mathbb{F}_{q}$ used to represent the sequence number (SEQ) and the CRC, respectively, and $\ell$ is the payload. SEQ and CRC form part of the header of networkcoded packets [13]. The CRC is used to protect the data, the coefficients involved in the linear combination, and SEQ.

Now, $P_{R}(\gamma \mid i, g, q)$ represents the pmf of the number $\gamma$ independent linear combinations among the $i$ received, and which is given by

$$
P_{R}(\gamma \mid i, g, q)=\frac{\mu(i, g, \gamma, q)}{q^{i g}}
$$

where $\mu\left(n_{1}, k_{1}, r_{1}, q\right)$ [14] is the number of matrices in $\mathbb{F}_{q}^{n_{1} \times k_{1}}$ with rank equal to $r_{1}$

$$
\mu\left(n_{1}, k_{1}, r_{1}, q\right)=\left[\begin{array}{c}
k_{1} \\
r_{1}
\end{array}\right]_{q} \sum_{i=0}^{r_{1}}(-1)^{\left(r_{1}-1\right)}\left[\begin{array}{c}
r_{1} \\
i
\end{array}\right]_{q} q^{n_{1} i+\left({ }^{r_{1}-i}\right)}
$$

and $\left[\begin{array}{l}n_{1} \\ k_{1}\end{array}\right]_{q}$ is the Gaussian coefficient [14] given by

$$
\left[\begin{array}{l}
n_{1} \\
k_{1}
\end{array}\right]_{q}= \begin{cases}1 & k_{1}=0 \\
\frac{\left(q^{n_{1}}-1\right)\left(q^{n_{1}-1}-1\right) \ldots\left(q^{n_{1}-k_{1}+1}-1\right)}{\left(q^{k_{1}}-1\right)\left(q^{k_{1}-1}-1\right) \ldots(q-1)} & k_{1}>0\end{cases}
$$

Assume now that the average SNR for Scenario $s=1, \ldots, 3$ when receiving $\gamma$ independent coded packets in $\mathbb{F}_{q}$ is given by $\operatorname{SNR}_{s}(\gamma, q)$, the average SNR accounting for the transition probability distribution $f(\varepsilon)$ is deduced from (4) and (6) and is expressed as

$$
\operatorname{SNR}_{s}(q)=\sum_{\gamma=0}^{n} \operatorname{SNR}_{s}(q, \gamma) P_{\Gamma}(\gamma \mid g, n, q) .
$$

When considering NC-SDC or NC-MDC-T, a closed-form expression for $\operatorname{SNR}_{s}(\gamma, q)$ may be obtained. In the case of NC-MDC-F, such expression is more complex to obtain. The following section characterizes the average SNR for various $f(\varepsilon)$ and sizes of the Galois field using (9).

\section{Simulation Results}

Simulations are performed with $k=6$ and $n=9$. The source generates vectors of $k$ independent and identically distributed Gaussian samples with zero-mean and variance $\sigma^{2}=1$. Quantization with $q \in\{7,17,31,61\}$ outputs are considered, leading to quantization indexes in $\mathbb{F}_{q}$. The network matrix $A$ is chosen at random in the corresponding Galois field to simulate the effect of network coding. Simulation results are averaged over 1000 realizations of the source and of the network matrix $A$.

\section{A. Performance as a function of the number of lost packets}

First, the average SNR resulting from the reconstruction of the transmitted message is drawn as a function of the number of lost packets for various sizes of the Galois field used in the quantization and $\mathrm{NC}$ operations. These curves correspond to the representation of $\sum_{\gamma} \operatorname{SNR}_{s}(q, \gamma) P_{R}(\gamma \mid i, g, q)$ with the number of lost packets being $n-i$. The potential rank 
deficiency is thus taken into account, but not the effect of transmission errors.

Figures 4 and 5 represent the SNR for NC-MDC-F, NCMDC-T, and NC-SDC as a function of the field size. For NC-SDC, $n$ network-coded packets containing $k$ independent packets are transmitted (the redundancy is almost the same as in the two first scenarios, only the size of the header is somewhat reduced due to the reduced number of independent packets). As stated in the introduction, Figures 4 and 5

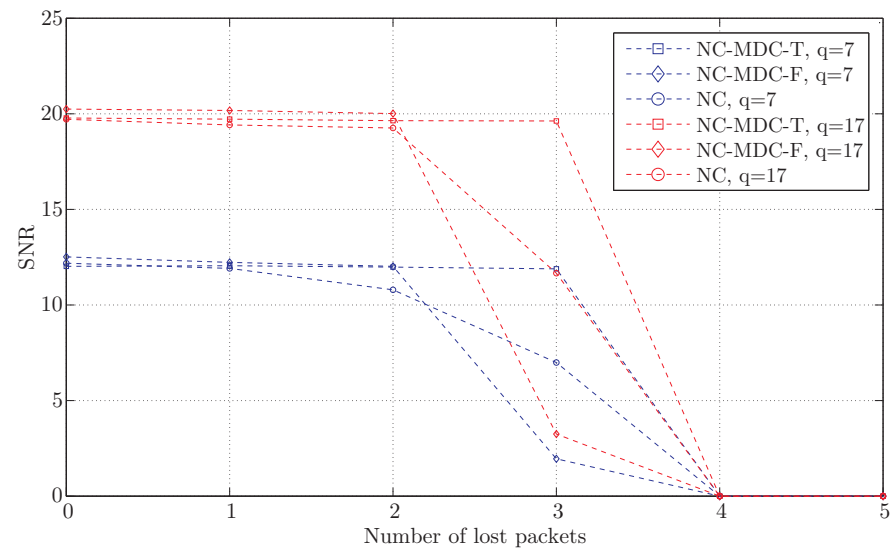

Fig. 4. SNR as a function of the number of lost packets and $q$, for $q=7$ and $q=17$. NC-MDC-F (diamond), NC-MDC-T (square), and NC-SDC (circle)

show that NC-MDC-F can mitigate part of the quantization noise, providing better results than the NC-MDC-T when the BSC transition probability is small. NC-MDC-T provides an increased robustness to packet losses compared to NC-MDCF.

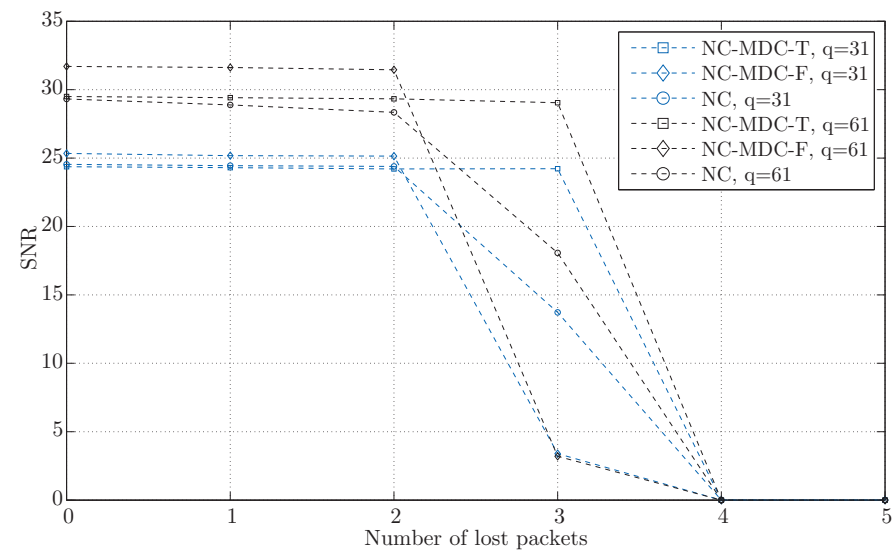

Fig. 5. SNR as a function of the number of lost packets and $q$, for $q=31$ and $q=61$. NC-MDC-F (diamond), NC-MDC-T (square), and NC-SDC (circle)

Figures 4 and 5 also show a degradation of the SNR due to the rank deficiency, even if enough packets have been received. When not enough packets are received, the SNR is null.

\section{B. Average SNR as a function of $\varepsilon$}

In this case the average SNR is evaluated as a function of the channel transition probability $\varepsilon$, with $q=31$. Figure 6 represents the SNR as a function of $\varepsilon$ when $\varepsilon$ is between $10^{-6}$ and $10^{-1}$ for packets with payloads of length $\ell=100$ symbols. NC-MDC-F outperforms NC-SDC for values of $\varepsilon$ in $\left[0,1.5 \times 10^{-4}\right]$, but for larger values of $\varepsilon$ the average SNR with NC-SDC is larger.

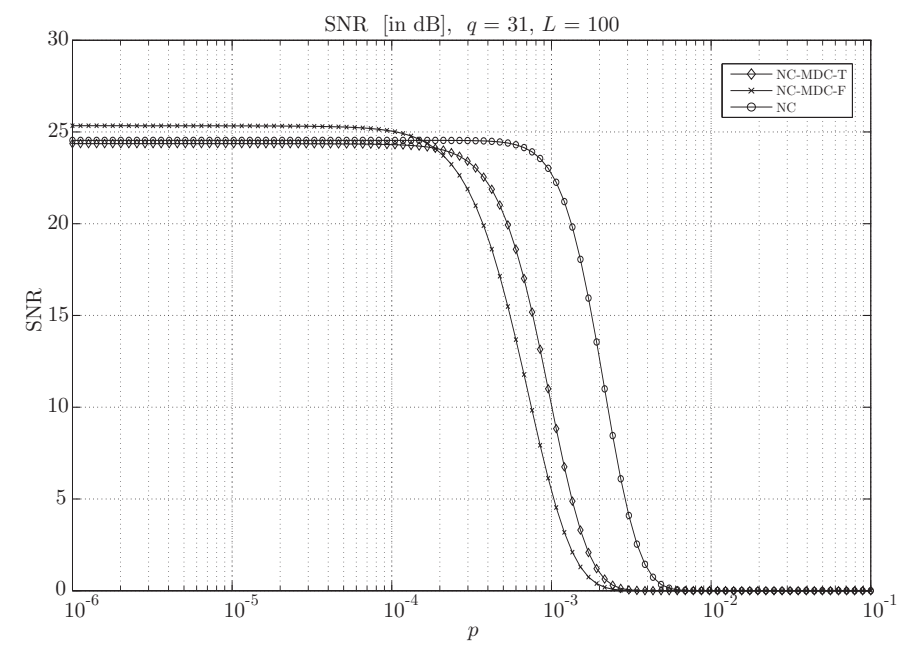

Fig. 6. Average SNR as a function of the transition probability $\varepsilon$

\section{Average SNR for various distributions of the transition probability}

In this case we study the received signal quality for two different pdfs of the transition probability $\varepsilon$. The uniform distribution

$$
f_{\mathrm{U}}(\varepsilon)=\left\{\begin{array}{cc}
\frac{1}{a} & 0 \leq \varepsilon \leq a \\
0 & \text { elsewhere }
\end{array}\right.
$$

and the exponential distribution

$$
f_{\mathrm{E}}(\varepsilon)= \begin{cases}\frac{\ln (10) 10^{-\varepsilon}}{10^{-a}-10^{-b}} & a \leq \varepsilon \leq b \\ 0 & \text { elsewhere }\end{cases}
$$

are considered.

Using (10), (4) becomes

$P_{C}(i \mid g, n, q)=\frac{1}{a}\left(\begin{array}{c}n \\ i\end{array}\right) \int_{0}^{a}(1-\varepsilon)^{i L}\left(1-(1-\varepsilon)^{L}\right)^{n-i} d \varepsilon$.

Using (11), (4) becomes

$$
\begin{gathered}
P_{C}(i \mid g, n, q)= \\
\frac{\ln (10)}{10^{-a}-10^{-b}}\left(\begin{array}{c}
n \\
i
\end{array}\right) \int_{a}^{b} 10^{-\varepsilon}(1-\varepsilon)^{i L}\left(1-(1-\varepsilon)^{L}\right)^{n-i} d \varepsilon .
\end{gathered}
$$

The average SNR is computed using (9) after substituting (12) or (13) in (3).

With NC-MDC-F and NC-MDC-T, the number of mixed packets is $g=n$ and with NC, it is $g=k$. Figure 7 represents the expected SNR for the presented techniques for various field sizes $q=\{7,17,31,61\}$ using the uniform pdf for the transition probability as a function of $a$, the upper bound of the support of the uniform pdf. 
Similarly, Figure 8 represent the expected SNR for the exponential pdf for the transition probability with $a=10^{-10}$ as a function of $b$, from $10^{-6}$ to $10^{-1}$.

In both cases, when there are not too many users with bad channel characteristics, NC-MDC-F outperforms NC-MDC-T, which itself outperforms NC. When the proportion of users with bad channel gets larger $\left(a>3.10^{-4}\right.$ for the uniform pdf and $b>3.10^{-4}$ for the exponential pdf), NC-SDC becomes better.

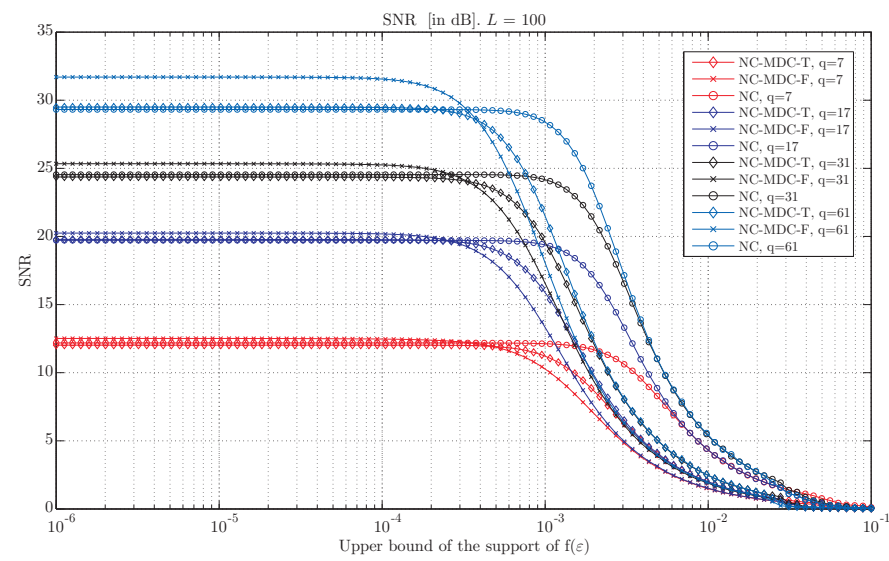

Fig. 7. Average SNR for the Uniform Distribution as a function of the upper bound of the support of the pdf of transition probability $f(\varepsilon)$

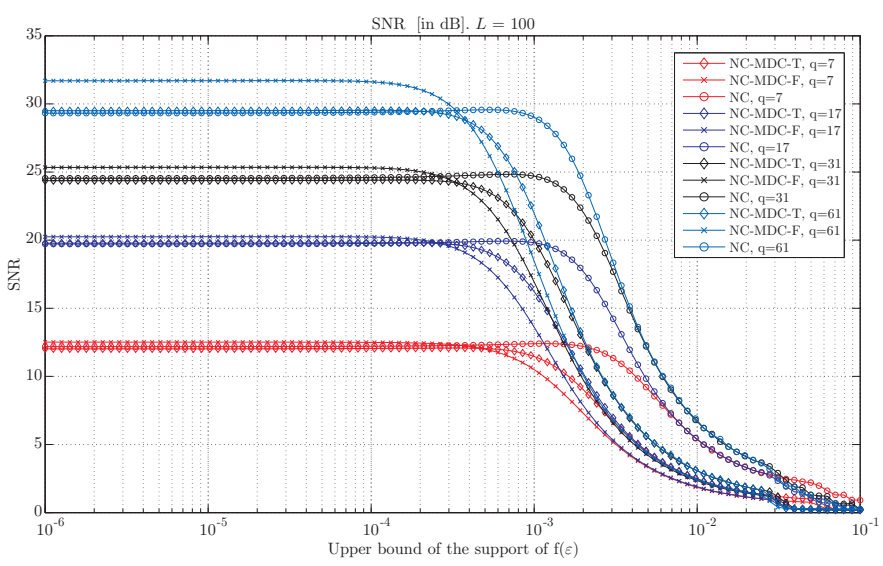

Fig. 8. Average SNR for the Exponential Distribution as a function of the upper bound of the support of the pdf of the transition probability $f(\varepsilon)$

\section{CONCLUSIONS AND PERSPECTIVES}

This paper considers the effects of the field size and channel conditions in the average signal quality received, in the scenario where a single source multicasts data to a set of users experiencing various channel conditions, using $\mathrm{NC}$ or a combination of MDC and NC.

Packets are assumed to be received at the output of a BSC which transition probability $\varepsilon$ is taken as random according to some pdf $f(\varepsilon)$. The performance of the three schemes largely depends on $f(\varepsilon)$. When a user is likely to have good channel conditions, NC-MDC-F provides the best results. When his channel conditions may be quite bad, then plain NC-SDC becomes better.

In future work, a distribution of the number of noisy packets obtained by the various receivers may be introduced to have a more realistic description of packet reception in wireless ad-hoc networks. One may also consider the distribution of channel SNR as a function of the user location when considering a cellular network. The effect of relays has also to be better taken into account.

\section{ACKNOWLEDGMENTS}

This work has been partly supported by DIM-LSC NC2, DIM-LSC SWAN. Michel Kieffer was partly supported by the Institut Universitaire de France.

\section{REFERENCES}

[1] M. Ghanbari, "Two-layer coding of video signals for vbr networks," IEEE J Select Areas Commun, vol. vol. 7, no. 5, pp . 771-781, June 1989.

[2] V. K. Goyal, "Multiple description coding: Compression meets the network," IEEE Signal Processing Mag, vol. vol. 18, no. 5, pp. 74-93, 2001.

[3] X. W. M. Shao and N. Sarshar, "Rainbow network flow with network coding," in Proc. of the fourth Workshop on Network Coding, Theory and applications, Netcod, Hong Kong, 2008.

[4] R. Ahlswede, N. Cai, S.-Y. R. Li, and R. W. Yeung, "Network information flow," IEEE Trans. on Information Theory, vol. 46, Jul 2000.

[5] Z. Li, B. Li, D. Jiang, and L. C. Lau, "On achieving optimal throughput with network coding," IEEE INFOCOMM, vol. vol. 3, pp $2184-2194$ 2005.

[6] T. Ho, M. Medard, R. Koetter, D. R. Karger, M. Effros, J. Shi, and B. Leong, "A random linear network coding approach to multicast," IEEE Transactions on Information Theory, vol. 52, no. 10, pp. 44134430, October 2006.

[7] J. Walsh and S. Weber, "A concatenated network coding scheme for multimedia transmission," in Proc. of the fourth Workshop on Network Coding, Theory and applications, Netcod, Hong Kong, 2008.

[8] H. Park, N. Thomos, and P. Frossard, "Transmission of correlated information sources with network coding," in EUSIPCO 2010, Aalborg, Denmark, August 2010.

[9] L. Iwaza, M. Kieffer, L. Liberti, and k. Al-Agha, "Joint decoding of multiple-description network-coded data," in Proc. of the international symposium on Network Coding, Netcod, Beijing, 2011.

[10] R. Fourer and D. Gay, The AMPL Book. Duxbury Press, 2002.

[11] ILOG CPLEX 11.0 User's Manual.

[12] C. E. Shannon, "A mathematical thoery of communication," Bell Syst. Tech. J., 1948.

[13] P. A. Chou, Y. Wu, and K. Jain, "Practical network coding," in Proc. 41st Annual Allerton Conference on Communication, Control, and Computing, 2003.

[14] J. H. van Lint and R. M. Wilson, A Course in Combinatorics. Cambridge University Press, Cambridge, UK, 1992. 